\title{
Stellar population analysis on local infrared-selected galaxies
}

\author{
X. Y. Chen ${ }^{1,2}$, Y. C. Liang ${ }^{1}$, F. Hammer ${ }^{3}$, Y. H. Zhao ${ }^{1}$, and G. H. Zhong ${ }^{1,4}$ \\ 1 National Astronomical Observatories, Chinese Academy of Sciences, A20 Datun Road, 100012 Beijing, PR China \\ e-mail: [chenxy;ycliang]@bao.ac.cn \\ 2 Graduate School of the Chinese Academy of Sciences, 100049 Beijing, PR China \\ 3 GEPI, Observatoire de Paris-Meudon, 92195 Meudon, France \\ 4 Department of Physics, Hebei Normal University, 050016 Shijiazhuang, PR China
}

Received 17 July 2008 / Accepted 25 November 2008

\section{ABSTRACT}

\begin{abstract}
Aims. We study the stellar population of local infrared galaxies, which contain star-forming galaxies, composite galaxies, LINERs, and Seyfert 2s. We also want to find whether infrared luminosity and spectral class have any effect on their stellar populations. Methods. The sample galaxies are selected from the main galaxy sample of SDSS-DR4 and then cross-correlated with the IRASPSCz catalog. We fit our spectra (stellar absorption lines and continua) using the spectral synthesis code STARLIGHT based on the templates of simple stellar population and the spectra of star clusters.

Results. Among the 4 spectral classes, LINERs present the oldest stellar populations, and the other 3 subsamples all present substantial young and intermediate age populations and very few old populations. The importance of young populations decreases from starforming, composite, Seyfert 2 to LINER. As for the different infrared luminosity bins, ULIGs \& LIGs $\left(\log \left(L_{\mathrm{IR}} / L_{\odot}\right) \geq 11\right)$ present younger populations than do starbursts and normal galaxies; however, the dominant contributors to mass are old populations in all sample galaxies. The fittings also give consistent results by using the spectra of star clusters with different ages and metallicities as templates. The dominated populations in star-forming and composite galaxies are those with metallicity $Z=0.2 Z_{\odot}$, while LINERs and Seyfert 2s are more metal-rich. The normal galaxies are more metal-rich than the ULIGs \& LIGs and starbursts for the starforming galaxies within different infrared luminosity bins.

Additionally, we compare some synthesis results with other parameters obtained from the MPA/JHU catalog. First we find that the stellar and nebular extinctions are correlated, and the ionized gas suffers twice as much extinction as stars. Second we confirm that $D_{n}(4000)$ is a much better age indicator than $H \delta_{A}$. Following the evolution of galaxies, $D_{n}(4000)$ monotonously varies. Finally we investigate some relationships between mean stellar age, mean stellar metallicity, and nebular metallicity for the subsample of star-forming galaxies. In star-forming galaxies, the nebular metallicity $Z_{\text {neb }}$ is correlated with the light-weighted mean stellar age $\left\langle\log t_{*}\right\rangle_{\mathrm{L}}$ in an intermediate strength, and $Z_{\text {neb }}$ is weakly correlated with the mass-weighted mean stellar metallicity $\left\langle Z_{*}\right\rangle_{\mathrm{M}}$.
\end{abstract}

Key words. galaxies: evolution - infrared: galaxies - galaxies: stellar content

\section{Introduction}

Infrared galaxies are objects that emit most of their energy in the infrared ( 5-500 $\mu \mathrm{m})$ (Sanders \& Mirabel 1996). They are one of the most important discoveries from extragalactic observations at mid- and far-infrared wavelengths. The overwhelming majority of the extragalactic objects detected in the Infrared Astronomical Satellite (IRAS) survey are late-type spiral galaxies, while elliptical and S0 galaxies are rarely detected (Soifer 1987). With the higher sensitivity and spatial resolution than IRAS, the ISO satellite moves the studies of luminous infrared galaxies to $z \geq 0.5$ from local (Elbaz et al. 1999, 2002; Flores et al. 1999; Liang et al. 2004; Zheng et al. 2004; Hammer et al. 2005, and references therein). The cosmic infrared background resolved by ISO mid-infrared camera (ISOCAM) shows that the co-moving density of infrared light due to the luminous IR galaxies was more than 40-70 times higher at $z \sim 1$ than today (Elbaz et al. 2002). And this evolution is mainly driven by the luminous infrared starburst galaxies, in which the star formation rate runs up to $100 M_{\odot} \mathrm{yr}^{-1}$ (Flores et al. 1999). The successful launch of the Spitzer Space Telescope opened a new exciting window on the deep infrared universe. Spitzer operates between 3.6 and $160 \mu \mathrm{m}$ with better sensitivity and spatial resolution than for IRAS and ISO, and provides new opportunities for determining the IR properties of galaxies in the general context of cosmic evolution (Le Floc'h et al. 2005; Zheng et al. 2007; Papovich et al. 2007, and references therein). The future Herschel will also improve much of our knowledge about infrared galaxies. Therefore, infrared-detected galaxies are one of the most interesting objects in the Universe and are related to the major star-forming process. The local infrared galaxies have the advantage of being studied in details with properties, such as stellar populations, since their high-quality optical spectra could be obtained in the modern digital sky survey, e.g. the Sloan Digital Sky Survey (SDSS).

Understanding the overall stellar population in galaxies is a crucial tool for unveiling the star formation and evolution of galaxies. As spectra of galaxies record information about age and metallicity distributions of its stellar populations, many works have been generated on stellar population analysis through spectra of galaxies, using the approach called stellar population synthesis. The first attempts were the empirical population synthesis (Faber 1972), in which the observed spectrum of a galaxy is reproduced by a combination of spectra of individual stars or star clusters of different ages and metallicities from a library. More recent models have been based on the evolutionary population synthesis technique (Tinsley 1978; Bruzual 1983; Charlot et al. 1996; Bruzual \& Charlot 2003), in which the main 
adjustable parameters could be the stellar initial mass function, the star formation rate, and the chemical enrichment. The reproduced simple stellar populations (SSP) with different ages and metallicities from the evolutionary population synthesis model could be used to analyze the stellar populations of the galaxies.

Some research has taken on analysis of stellar populations in star-forming galaxies and starburst galaxies from the optical band observations, most of which conclude that H II galaxies are age-composite stellar systems (Schmitt et al. 1996; Raimann et al. 2000; Cid Fernandes et al. 2003; Kong et al. 2003; Westera et al. 2004). Hammer et al. (2001) used the spectra of star clusters to analyze the stellar populations of distant luminous blue compact galaxies at redshift $0.4<z<1$ and find that they have included some old populations.

Moreover, some works have been performed on ultraviolet (UV) and near infrared bands. Based on International Ultraviolet Explore (IUE) UV spectra, Bonatto et al. (2000) discuss the stellar population of galaxies with enhanced star formation, which are at distances corresponding to radial velocities in the range of $5000-16000 \mathrm{~km} \mathrm{~s}^{-1}$. They find that young stellar populations (age < $500 \mathrm{Myr}$ ) are the main contributors except for galaxies with a red spectrum arising from the intermediate (age 1-2 Gyr) and old-age populations. Riffel et al. (2008) have studied the central stellar populations of four starburst galaxies through near-infrared spectroscopy and find that the dominate population was a 1 Gyr old with solar metallicity component in all galaxies.

Furthermore, some research was applied to AGNs. Cid Fernandes et al. present a series of studies that discussed the stellar populations of low-luminosity active galactic nuclei (LLAGN) (Cid Fernandes et al. 2004a, 2005a; González Delgado et al. 2004). They find that young stars contribute very little to the optical continuum, while intermediateage stars contribute significantly; and most of the strong-[OI] LLAGNs predominantly have old stellar population. Besides that, some works have been dedicated to the stellar populations of Seyfert 2s, and they confirm that most Seyfert $2 \mathrm{~s}$ contain a young hot stellar population (Schmitt et al. 1999; Storchi-Bergmann et al. 2000; González Delgado et al. 2001; Joguet et al. 2001; Cid Fernandes et al. 2004b). Boisson et al. (2000) performed stellar population synthesis on a sample of 12 galaxies (Boisson et al. 2004, studied 5 more) with different levels of activity, and they find different populations for different activity types: the starburst galaxies present the youngest populations of the sample; the Seyfert 2s also have young stellar population, less intense than in the starbursts, but metal rich; while LINERs show the oldest populations, metal rich, with little star formation still going on. But their sample was small, so a much larger sample from the modern digital sky survey is needed to verify these results.

To our knowledge, no work has been specifically dedicated to the stellar population analysis of local infrared-selected galaxies. In this paper, we utilize the data from cross identifying between SDSS and IRAS to study the stellar population of local (with median value of redshift $\sim 0.03$ ) infraredselected galaxies by using a stellar population synthesis program called STARLIGHT (Cid Fernandes et al. 2005b). With the high-quality SDSS optical spectra and the IRAS observations, this could be the largest local sample selected from infrared to date for studying their stellar populations from the full spectrum fitting pixel by pixel. Moreover, as our sample contains diverse spectral classes (star-forming galaxies, composite galaxies, LINERs, and Seyfert 2s) and spans a broad range of infrared luminosity, we can compare the properties among these different classes.

This paper is organized as follows. We present the sample selection and the classifications in Sect. 2. In Sect. 3 we describe the methods of the spectral synthesis and the results of fitting the spectra of galaxies in different classes and different bins of infrared luminosity. In Sect. 4, we present the relations of some property parameters from emission lines, continua of galaxies, and some from the spectral synthesis fittings on the spectra. Summary and conclusions are given in Sect. 5. Throughout this paper we assume the following cosmological parameters: $\Omega_{\mathrm{M}}=0.3, \Omega_{\Lambda}=0.7$, and $H_{0}=70 \mathrm{~km} \mathrm{~s}^{-1} \mathrm{Mpc}^{-1}$.

\section{Sample selection and classification}

Our sample was selected from the main galaxy sample of the Sloan Digital Sky Survey (SDSS) DR4 database and then was cross-correlated with the IRAS Point Source Catalog (PSCz).

The SDSS-DR4 includes over 800000 fiber spectra of objects selected based on the five bands $(u, g, r, i$, and $z)$ imaging (Fukugita et al. 1996; Smith et al. 2002; Strauss et al. 2002). Each fiber is 3" in diameter (Bernardi et al. 2003; Greene et al. 2005, 2006; Adelman-McCarthy et al. 2006), corresponding to $\sim 1.8 \mathrm{kpc}$ at $z=0.03$ (the median redshift of our sample, see below and Fig. 1). The observed-frame spectral wavelength range is 3800-9200 $\AA$. The instrumental resolution of the spectra is $\lambda / \Delta \lambda \approx 1800$ equivalent to Gaussian $\sigma_{\text {inst }} \approx 70 \mathrm{~km} \mathrm{~s}^{-1}$ (Heckman et al. 2004; Greene et al. 2005, 2006).

In particular, we selected all galaxies with redshift less than 0.5 and redshift confidence greater than 0.9 from SDSS DR4. After that these objects were cross-identified with PSCz using a 5" matching radius. The IRAS PSCz survey is a redshift survey of about 15000 galaxies detected, after supplements and corrections (Saunders et al. 2000), containing 18351 objects with $f_{60}>0.6 \mathrm{Jy}$, covering $84 \%$ of the full sky. The optical positions of PSCz objects were used to assure the reliability of the identification. As the PSCz objects mostly have redshift less than 0.1 (Saunders et al. 2000), the redshift distribution of our sample objects is almost truncated after a redshift of 0.1 .

After cross-identification, 1097 target objects were obtained. To improve the accuracy of the classification of our sample objects, we excluded 34 objects without emission-line flux measured in the MPA/JHU catalog. Meanwhile we excluded another 65 objects with low emission-line signal-to-noise ratio $(\mathrm{S} / \mathrm{N})$ according to their $\mathrm{H} \beta, \mathrm{H} \alpha$, and [NII] $\lambda 6583$ lines detected at lower than $5 \sigma$, and [OIII] $\lambda 5007$ line detected at lower than $3 \sigma$. After this cut, 998 sources are left.

To ensure the spectra were taken at the centers of the galaxies (within a radius of $2^{\prime \prime}$ from the center), we examined the positions of fibers for all the sample objects. We found that there were 49 galaxies with the fibers not located at the centers. After removing them, we have 949 objects left in total.

To do the spectral synthesis fittings, 100 objects were further ejected because their mask spectra were problematic, in which almost half of the spectra in wavelength were masked (see more details in Sect. 3.1). Finally, 849 objects were left as our working sample for a stellar population analysis.

The redshifts of the whole sample of 849 galaxies range from 0.002 to 0.24 , with a median of 0.03 , which is presented in Fig. 1 (left column). In this work, we used two methods (by emissionline ratios and by infrared luminosity) to classify the sample galaxies into several subgroups as shown below. 


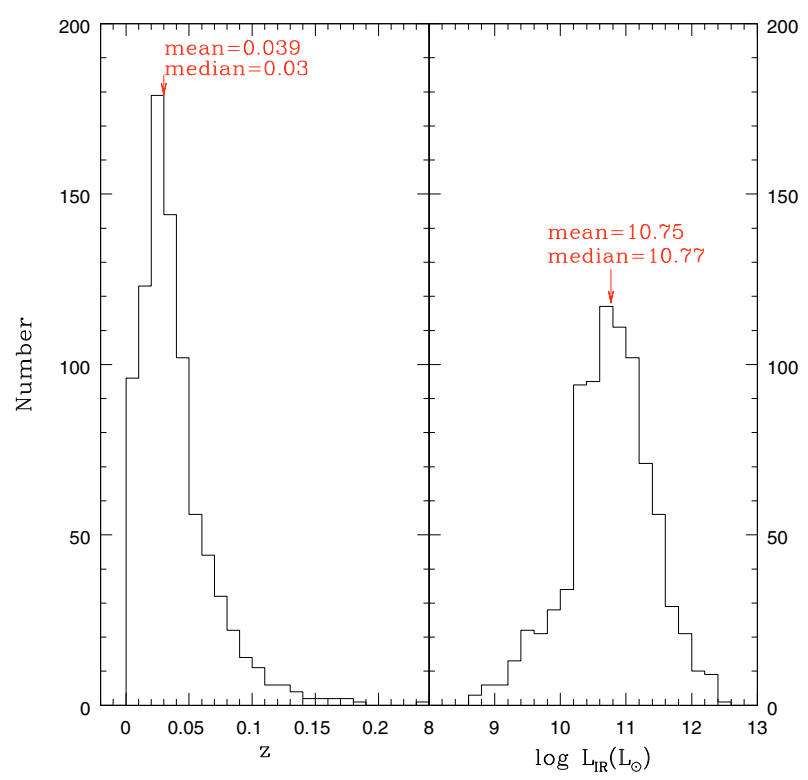

Fig. 1. The distributions of redshift (left) and infrared luminosity (right) for our sample of 849 infrared-selected galaxies.

\subsection{Classification by emission-line ratios}

The first method to classify the samples is using an emissionline diagnostic diagram. Namely, we classified the 849 sources as star-forming galaxies, composite galaxies, LINERs, and Seyfert $2 \mathrm{~s}$ according to the emission-line flux ratio diagnostic diagram (Fig. 2, Baldwin et al. 1981, BPT; Veilleux \& Osterbrock 1987; Kauffmann et al. 2003a; Kewley et al. 2001). First, we separated star-forming galaxies, composite galaxies, and AGNs by Eq. (1) (Kewley et al. 2001, Fig. 2) and Eq. (2) (Kauffmann et al. 2003a, Fig. 2). The composite galaxies refer to objects whose spectra contain significant contributions from both AGN and star formation (Brinchmann et al. 2004; Kewley et al. 2006). Then we divided AGNs into LINERs and Seyfert 2 s by Eq. (3) (Shuder et al. 1981). The fluxes of these emission lines were derived from the MPA/JHU catalog. We finally obtained 419 star-forming galaxies, 326 composite galaxies, 69 LINERs, and 35 Seyfert $2 \mathrm{~s}$ (Table 1):

$$
\begin{aligned}
& \log \left(\frac{[\mathrm{OIII}] \lambda 5007}{\mathrm{H} \beta}\right)=\frac{0.61}{\log ([\mathrm{NII}] \lambda 6583 / \mathrm{H} \alpha)-0.47}+1.19, \\
& \log \left(\frac{[\mathrm{OIII}] \lambda 5007}{\mathrm{H} \beta}\right)=\frac{0.61}{\log ([\mathrm{NII}] \lambda 6583 / \mathrm{H} \alpha)-0.05}+1.3, \\
& {[\mathrm{OIII}] \lambda 5007 / \mathrm{H} \beta=3 .}
\end{aligned}
$$

\subsection{Classification by infrared luminosity}

We calculated the far-infrared luminosity $L_{\mathrm{FIR}}$ of the sample galaxies from the nominal flux densities, $f_{v}(60 \mu \mathrm{m})$ and $f_{v}(100 \mu \mathrm{m})$ in the IRAS catalogs (Helou et al. 1988; Sanders $\&$ Mirabel 1996), and the redshifts given by SDSS, and then we converted it to the total infrared luminosity $L_{\mathrm{IR}}(1-1000 \mu \mathrm{m}$, Calzetti et al. 2000; Cao et al. 2006; Wang et al. 2006; Wang 2008; Chen et al. 2008) according to the following formulas:

$$
\begin{aligned}
& F_{\mathrm{FIR}}=1.26 \times 10^{-14}\left\{2.58 f_{60}+f_{100}\right\}\left[\mathrm{W} \mathrm{m}^{-2}\right], \\
& L_{\mathrm{FIR}}=4 \pi D_{\mathrm{L}}^{2} F_{\mathrm{FIR}}\left[L_{\odot}\right], \\
& L_{\mathrm{IR}}\left(1 \sim 1000 \mu_{\mathrm{m}}\right)=1.75 L_{\mathrm{FIR}} .
\end{aligned}
$$

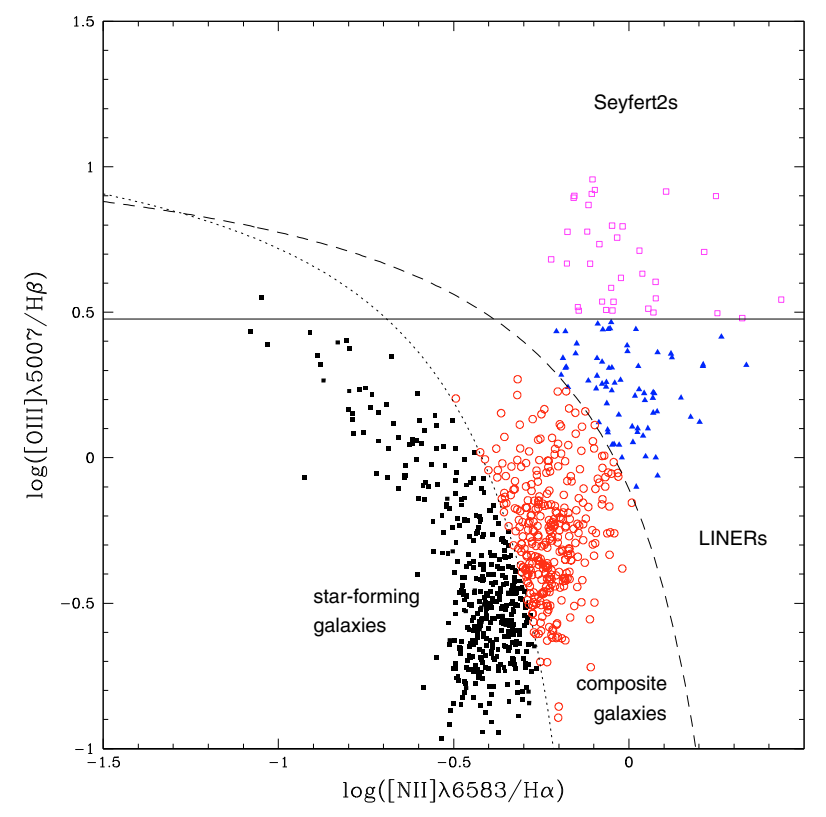

Fig. 2. The emission-line flux ratio diagnostic diagram (i.e. BPT, Baldwin et al. 1981) for our full sample (849), in which we plot the ratio of $[\mathrm{OIII}] / \mathrm{H} \beta$ versus the ratio of $[\mathrm{NII}] / \mathrm{H} \alpha$. The dotted curve defined by Kauffmann et al. (2003a) and the dashed curve defined by Kewley et al. (2001) show the separation among star-forming galaxies, composite galaxies, and AGNs. The solid line defined by Shuder et al. (1981) shows the separation between LINERs and Seyfert $2 \mathrm{~s}$.

The infrared luminosity of the whole sample of 849 galaxies spans a range of $\log \left(L_{\mathrm{IR}} / L_{\odot}\right)=8.65-12.53$, with a median of 10.77 , and we show the distribution of infrared luminosity in Fig. 1 (right column).

The second classification method was in accordance with their infrared luminosities. Namely, we divided our 849 objects into 3 subsamples according to their infrared luminosity (Elbaz et al. 2002). As a result, there are 299 ULIGs and LIGs $\left(L_{\mathrm{IR}} / L_{\odot}>10^{11}\right), 451$ starbursts $\left(10^{10}<L_{\mathrm{IR}} / L_{\odot}<10^{11}\right)$, and 99 normal galaxies $\left(L_{\mathrm{IR}} / L_{\odot}<10^{10}\right)$ in our sample. But the number of the ULIGs is very small (there are only 20 ULIGs in 849 objects).

We divided each of the spectral classes from emission-line ratios (i.e. star-forming galaxies, composite galaxies, LINERs, and Seyfert 2s) further into 3 infrared luminosity bins as above. The related numbers of the objects are given in Table 1 .

\section{Spectral synthesis}

In this section, we fit the spectral absorptions and continua of the sample galaxies to study their stellar populations by using the software STARLIGHT ${ }^{1}$ (Cid Fernandes et al. 2005b, see details below). We downloaded the spectra in a one-dimensional FITS format ${ }^{2}$, and they have been sky-subtracted, then the telluric absorption bands were removed before the wavelength and spectrophotometric were calibrated (Stoughton et al. 2002). We corrected the foreground Galactic extinction using the reddening maps of Schlegel et al. (1998) and then shifted the spectra to the rest frame. To obtain the general view of the stellar populations of galaxies in different spectral classes and to improve the $\mathrm{S} / \mathrm{N}$ of the spectra, we combined the spectra of all the objects

\footnotetext{
1 http://www.starlight.ufsc.br

2 website: http://das.sdss.org/DR4-cgi-bin/DAS
} 
Table 1. Number of each subsample.

\begin{tabular}{|c|c|c|c|c|c|}
\hline Method & \multicolumn{4}{|c|}{ Emission-line diagram } & \multirow[t]{2}{*}{ Infrared luminosity bin } \\
\hline class & star-forming & composite & LINER & Seyfert 2 & \\
\hline total number & 419 & 326 & 69 & 35 & 849 \\
\hline ULIG \& LIG & 100 & 158 & 26 & 15 & 299 \\
\hline starburst & 250 & 152 & 32 & 17 & 451 \\
\hline normal & 69 & 16 & 11 & 3 & 99 \\
\hline
\end{tabular}

to be one spectrum in each of the spectral classes: star-forming galaxies, composite galaxies, LINERs, and Seyfert 2s. Likewise, to check the effect of infrared luminosity on stellar populations of galaxies, we took star-forming galaxies as representative and combined their spectra in each of the three infrared luminosity bins. We also did the same stellar population synthesis fittings on the individual spectrum of the 849 sample galaxies. The results will be discussed in Sect. 4.

\subsection{The method}

The software STARLIGHT was developed by Cid Fernandes and his colleagues to synthesize the stellar absorptions and continua of the sample galaxies (Cid Fernandes et al. 2005b, 2007; Mateus et al. 2006; Asari et al. 2007). It is a program to fit an observed spectrum $O_{\lambda}$ with a model $M_{\lambda}$ that adds up to $N_{*}$ SSPs with different ages and metallicities from the evolutionary synthesis models of Bruzual \& Charlot (2003, hereafter BC03). The line-of-sight stellar motions are modeled by a Gaussian distribution centered at velocity $v_{*}$ and broadened by $\sigma_{*}$. The fit is carried out with the Metropolis scheme (Cid Fernandes et al. 2001), which searches for the minimum $\chi^{2}=\Sigma_{\lambda}\left[\left(O_{\lambda}-M_{\lambda}\right) \omega_{\lambda}\right]^{2}$, where $\omega_{\lambda}^{-1}$ is the error in $O_{\lambda}$ except for masked points. Pixels that are more than $3 \sigma$ away from the rms $O_{\lambda}-M_{\lambda}$ are given zero weight by the parameter "clip".

To extract the SSP from BC03, we used the "Padova 1994" tracks (Alongi et al. 1993; Bressan et al. 1993; Fagotto et al. 1994a,b; Girardi et al. 1996) and a Chabrier (2003) initial mass function (IMF). Extinction was modeled as rising from the foreground dust, with the reddening law of Calzetti et al. (1994, CAL hereafter) with $R_{v}=3.1$. As mentioned by the manual of STARLIGHT that the reddening law of Cardelli et al. (1989, CCM hereafter) was not a good choice for ULIGs, we used CAL law. Moreover, we used a base of 45 SSPs, which included 15 different ages from 1 Myr to 13 Gyr (i.e. 1, 3, 5, 10, 25, 40, 100, 280, 640, $900 \mathrm{Myr}$ and 1.4, 2.5, 5, 11, $13 \mathrm{Gyr}$ ) at each of the three metallicities: $0.2,1$, and $2.5 Z_{\odot}$. All bases were normalized at $\lambda_{0}=4020 \AA$. Additionally, a power law stands for nonstellar component (Koski et al. 1978) $F_{v} \propto v^{\alpha}$, was added when we fit the spectra of AGNs (both LINERs and Seyfert 2s). We set the power-law index as $\alpha=-1.5$ (Cid Fernandes et al. 2004b). We will first describe the method for spectra synthesis. All spectra were sampled again in steps of $\Delta \lambda=1 \AA$ from 3700 to $8000 \AA$, and normalized by the median flux in the 4010 to $4060 \AA$ region. The $\mathrm{S} / \mathrm{N}$ in the normalization window spans a range of 1.1-86.1, with median value of 15.6. Then we compiled the mask-files for our sample. Besides emission line and sky line, we also excluded points that have nonzero mask spectra ${ }^{3}$ in SDSS, which are bad pixels or other artifacts. We furthermore excluded four other windows: $5870-5905 \AA$, to leap the $N a D \lambda \lambda 5890,5896$ doublet, which is from the interstellar medium; 6845-6945 $\AA$

\footnotetext{
${ }_{3}$ http://www.sdss.org/dr4/dm/flatFiles/spSpec.html\# specmask
}

and $7550-7725 \AA$, which are listed by BC03 as their bugs due to problems in these ranges in the STELIB library (Le Borgne et al. 2003); 7165-7210 A, which shows a systematic broad residual in emission as mentioned by Mateus et al. (2006). In addition, there were 100 objects (56 star-forming galaxies, 34 composite galaxies, 6 LINERs, and 4 Seyfert 2s) whose mask spectra were problematic, in which almost half of the spectra were masked. We do not fit these 100 spectra as mentioned in Sect. 2.

In the outputs of STARLIGHT, one of the most important parameters to present stellar population is the population vector $x$. The component $x_{j}\left(j=1, \ldots, N_{*}\right)$ represents the fractional contribution of the SSP with age $t_{j}$ and metallicity $Z_{j}$ to the model flux at the normalization wavelength $\lambda_{0}=4020 \AA$. Equivalently, another important parameter, the mass fraction $\mu_{j}$, has the similar meaning. We analyze the obtained values of these parameters.

\subsection{The results of fitting the combined spectra according to different spectral classes}

As discussed in Sect. 2.1, our sample can be divided into four subsamples by a diagnostic diagram of their emission-line ratios, i.e., star-forming galaxies, composite galaxies, LINERs, and Seyfert 2s. To clearly show their properties and to examine whether there is any difference in the stellar populations of different spectral classes, we combine all the spectra of the sample galaxies in each subsample, then we synthesize these combined spectra with improved $\mathrm{S} / \mathrm{N}$.

Figure 3 shows the spectral fitting results for our combined spectra of 419 star-forming galaxies (the four panels at top-left), 326 composite galaxies (the four panels at top-right), 69 LINERs (the four panels at bottom-left), and 35 Seyfert 2 s (the four panels at bottom-right).

For each of the subgroups, we plot the observed spectra, the synthesis spectra, the error spectra, and the residual spectra (the pure emission-line spectra) in the left two columns, and the light and mass fractions as functions of ages of each SSPs in the right columns. We can see that the error spectra are all very weak except the area around $\mathrm{H} \beta$, and the emission lines, especially the weak ones, appear much more clearly in the residual spectra.

Additionally, at the top right of each subgroup, we also list some other parameters, such as $\chi_{\lambda}^{2}$, i.e. the reduced $\chi^{2}$, the mean relative difference between synthesis and observed spectra $\Delta_{\lambda}$; the $\mathrm{S} / \mathrm{N}$ in the region of 4730-4780 $\AA$; the $V$-band stellar extinction; the velocity and the velocity dispersion. Again we confirm that our fit is good enough except for the area around $\mathrm{H} \beta$. As to the bad fit around $\mathrm{H} \beta$, which means that there is always a trough in the continuum around $\mathrm{H} \beta$, Asari et al. (2007) suggests that this could relate to calibrations in the STELIB library in this spectral range. The good fits around $\mathrm{H} \beta$ by using the spectra of star clusters instead presented in Sect. 3.6 confirm this. However, this problem around $\mathrm{H} \beta$ will not affect our general results much for the stellar populations of the galaxies.

To obtain a general view of the stellar populations of the sample galaxies, we arrange their stellar populations into three 

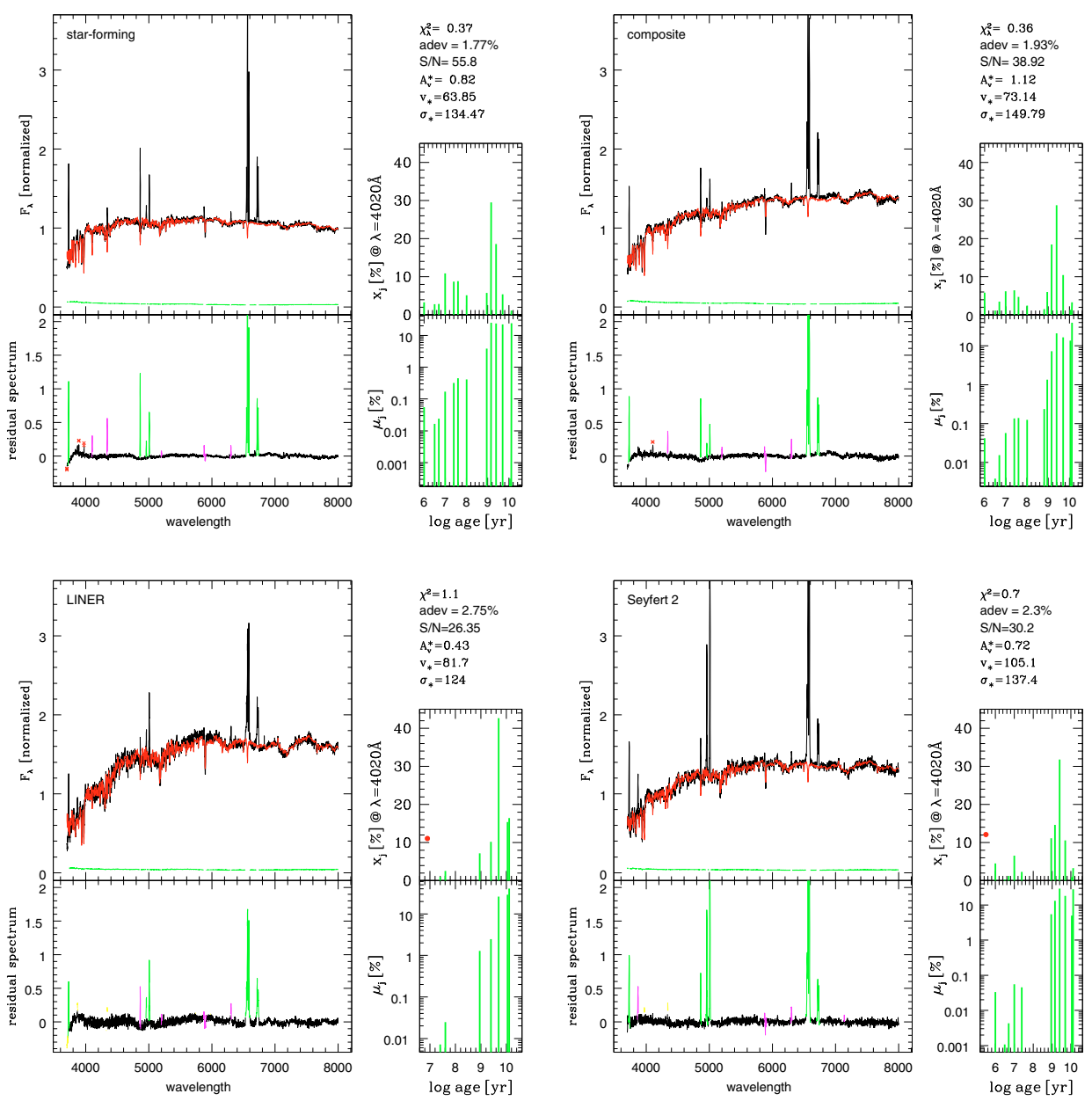

Fig. 3. The spectral synthesis results of the combined spectra of each spectral class by using 45 SSPs from BC03. The subsamples are star-forming galaxies (top-left four panels), composite galaxies (top-right four panels), LINERs (bottom-left four panels), and Seyfert 2s (bottom-right four panels). Top left: comparison of synthesis spectrum (red line) with the observed spectrum (black line), and green line shows the error spectrum; bottom left: the residual spectrum, green lines represent mask regions given by SDSS flag, magenta lines represent mask regions given by ourselves, and red crosses represent clip points judged by STARLIGHT code; top right: fraction of light at $\lambda_{0}$ associated to each of the 15 ages of SSPs used in our fits. The red points in this panel of Seyfert $2 \mathrm{~s}$ and LINERs represent the fraction of power-law; bottom right: fraction of mass (log-coordinate) as a function of the 15 ages of SSPs used in our fits. In the top right corner of each group, we list some parameters explained in text (please see the online color version for more details).

Table 2. Stellar populations in each subsample, i.e., the results of fitting the combined spectra by using 45 SSPs from BC03.

\begin{tabular}{cc|cccc|ccc}
\hline \hline & SSP & \multicolumn{4}{|c|}{ Emission-line diagram } & \multicolumn{3}{c}{ Star-forming in $L_{\text {IR }}$ bins } \\
& & star-forming & composite & LINER & Seyfert 2 & ULIG\&LIG & starburst & normal \\
\hline age & young & 41.4 & 30.2 & 3.4 & 15.1 & 51.3 & 32.6 & 40.4 \\
& intermediate & 57.5 & 65.2 & 56.4 & 68.5 & 44.4 & 63.4 & 59.6 \\
& old & 1.1 & 4.6 & 29.9 & 4.3 & 4.3 & 4.0 & 0.0 \\
& power law & & & 10.3 & 12.1 & & & \\
\hline$Z / Z_{\odot}$ & 0.2 & 78.5 & 84.8 & 40.4 & 44. & 71.9 & 76.9 & 42.7 \\
& 1.0 & 14.4 & 10.5 & 40.4 & 7.4 & 19.0 & 15.1 & 14.4 \\
& 2.5 & 7.1 & 4.7 & 8.9 & 36.5 & 9.1 & 8.0 & 42.9 \\
& power law & & & 10.3 & 12.1 & & & \\
\hline
\end{tabular}

age bins: old populations with age $\geq 1 \times 10^{10} \mathrm{yr}$, intermediate age populations with age between $6.4 \times 10^{8} \mathrm{yr}$ and $5 \times 10^{9} \mathrm{yr}$, and young populations with age $\leq 5 \times 10^{8}$ yr. Then we list the contributions of each age bin to the 4 combined spectra in percentage in Table 2 . The corresponding fractions for different infrared luminosity bins are also given, and we show the fractions of 3 metallicity bins. We should notice that these numbers are the percent fractions, which is a bit different from the $x_{j}$ component output by STARLIGHT directly. Combining Table 2 and the top right panels in each subgroup of Fig. 3, we find that star-forming galaxies, composite galaxies, and Seyfert 2 s contain substantial young and intermediate age populations, while old populations give very few contributions. As to LINERs, the dominant contributions come from old and intermediate age populations, while there are almost no young components. Therefore we conclude that LINERs present the oldest stellar populations among four 

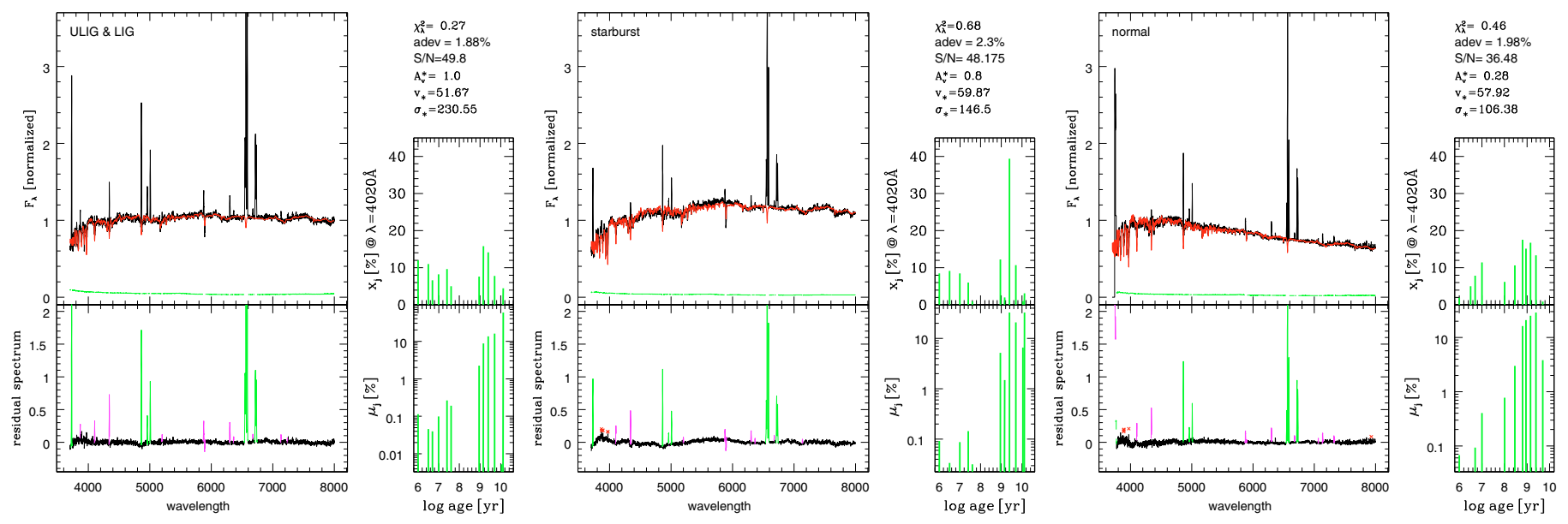

Fig. 4. Spectral fittings for the combined spectra of star-forming galaxies in each infrared luminosity bin by using 45 SSPs from BC03. The left four panels are for ULIGs \& LIGs $\left(\log \left(L_{\mathrm{IR}} / L_{\odot}\right)>11\right)$, the middle four panels are for starbursts $\left(10<\log \left(L_{\mathrm{IR}} / L_{\odot}\right)<11\right)$, and the right four panels are for normal galaxies $\left(\log \left(L_{\mathrm{IR}} / L_{\odot}\right)<10\right)$. All symbols are the same as in Fig. 3.

subsamples, and the star-forming galaxies have more younger populations than others. Besides that, we find the significance of young $\left(\leq 5 \times 10^{8}\right)$ populations decreases from star-forming, composite, Seyfert 2, to LINER. This gives credibility to our results, as Seyfert 2s and LINERs are well known to have their activities dominated by AGNs (or possibly shocks for LINERs), while starbursts are, in principle, with young stars. These results are consistent with Boisson et al. (2000, 2004), Kauffman et al. (2003a), González Delgado et al. (2004), Cid Fernandes et al. (2008), and Stasińaka et al. (2008).

Another phenomenon we noticed is that the dominant contributors to mass are all old stellar populations in four subsamples, and we speculate that it is due to the much quicker evolution of young massive stars than old ones. As mentioned in Sect. 3.1, we added a featureless continuum (power-law) in fitting spectra of LINERs and Seyfert 2 s, and this component contributes almost $10 \%$ to the light of galaxies (Table 2). For another test, we removed the power-law component of LINERs, making their young populations increase about $8 \%$. On the other hand, if we add a power law to composite galaxies, then the power law contributes little (about 3\%). There are some changes, but the dominant stellar populations are unchanged, which confirms our results.

The templates that we used in doing spectral synthesis are 45 in total, including 15 SSPs at each of the three metallicity grids $Z=0.2 Z_{\odot}, Z_{\odot}, 2.5 Z_{\odot}$. This could let us check the metallicity effect further, although the degeneracy of metallicity and age is popularly known. Then we obtained the contributions of each of the three metallicity grids by summing their contributions at different ages, which are given in Table 2 . It shows that the starforming and composite galaxies are dominated by the populations of $Z=0.2 Z_{\odot}$, meaning that most of their populations have metallicities even lower than $Z_{\odot}$, and LINERs and Seyfert $2 \mathrm{~s}$ are more metal-rich. The populations of $Z=Z_{\odot}$ significantly contribute to LINERs, which is consistent with the suggestion of Boisson et al. (2000, 2004), who comment that LINERs show the oldest populations in the sample, metal-rich, with little star formation still going on. The Seyfert $2 \mathrm{~s}$ show a significant contribution from the SSPS with $Z=0.2,2.5 Z_{\odot}$, which could be consistent with Cid Fernandes et al. (2004b), who suggest that the star formation history of Seyfert 2 nuclei is remarkably heterogeneous: young starbursts, intermediate-age, and old stellar populations all appear in significant and widely varying proportions.

\subsection{The results of fitting the combined spectra according to infrared luminosity}

To investigate whether the stellar populations of galaxies correlate at all with the infrared luminosity and to avoid the effects by spectral class, we specifically combine the spectra of starforming galaxies in three different infrared luminosity bins (as mentioned in Sect. 2.2), and fit their spectra. We list the spectral fitting results in Fig. 4.

Similar to Fig. 3, we get very weak error spectra and good residual spectra in left columns except areas around $\mathrm{H} \beta$. Moreover, in Table 2, we also list the contributions of each age bin to the combined spectra. Combining Table 2 and the light fraction distributions in Fig. 4, we can see that all subsamples contain both remarkable young and intermediate age populations and a few old populations, while ULIGs \& LIGs present the most significant fraction of young populations among three subsamples. The population with intermediate age is the dominant one in the starbursts and normals. As for the stellar populations in each grid of metallicities, the normal galaxies are more metal-rich than ULIGs \& LIGs and starbursts. The last two are dominated by the $Z=0.2 Z_{\odot}$ components.

\subsection{The spectral synthesis for combined spectra by using SSPS of $Z_{\odot}$ and $0.2 Z_{\odot}$}

To avoid the age-metallicity degeneracy and to further check the robustness of our estimations about the stellar populations of the sample galaxies in different age bins, we only adopt the $15 \mathrm{SSP}$ at each of $Z=Z_{\odot}$ and $Z=0.2 Z_{\odot}$ to re-do all the fittings on the 7 combined spectra studied above. The results are given in Tables 3 and 4, respectively. The way we arrange age bins are the same as before. The corresponding fractions for different infrared luminosity bins are also given. The dominant contributions have no obvious changes except the "old" populations increase (and the intermediate ones decrease) by some fractions, $\sim 5-15 \%$ in the $Z=Z_{\odot}$ case, and $\sim 10-30 \%$ in the $Z=0.2 Z_{\odot}$ case. The corresponding changing fractions are about $1-7 \%$ and $2-20 \%$ for star-forming galaxies in infrared luminosity bins. These could be understood simply because the old populations should increase their contributions to supply the lack of metal-rich components. Therefore, we believe that our estimates about the stellar populations of the sample galaxies are robust. 
Table 3. Stellar populations in each subsample, i.e., results of fitting the combined spectra by using $15 \operatorname{SSPs}\left(Z=Z_{\odot}\right)$ from BC03.

\begin{tabular}{cc|cccc|ccc}
\hline \hline & SSP & \multicolumn{4}{|c|}{ Emission-line diagram } & \multicolumn{3}{c}{ Star-forming in $L_{\text {IR }}$ bins } \\
& & star-forming & composite & LINER & Seyfert 2 & ULIG\&LIG & starburst & normal \\
\hline age & young & 38.6 & 33.5 & 0.1 & 12.0 & 50.6 & 31.4 & 31.8 \\
& intermediate & 55.6 & 57.4 & 43.4 & 54.3 & 39.3 & 65.0 & 66.9 \\
& old & 5.8 & 9.1 & 39.7 & 19.0 & 10.1 & 3.6 & 1.3 \\
& power law & & & 16.8 & 14.7 & & & \\
\hline
\end{tabular}

Table 4. Stellar populations in each subsample, i.e., results of fitting the combined spectra by using $15 \operatorname{SSPs}\left(Z=0.2 Z_{\odot}\right)$ from BC03.

\begin{tabular}{cc|cccc|ccc}
\hline \hline & SSP & \multicolumn{4}{|c|}{ Emission-line diagram } & \multicolumn{3}{c}{ Star-forming in $L_{\text {IR }}$ bins } \\
& & star-forming & composite & LINER & Seyfert 2 & ULIG\&LIG & starburst & normal \\
\hline age & young & 41.6 & 35.0 & 3.9 & 16.1 & 56.2 & 37.0 & 47.9 \\
& intermediate & 45.4 & 43.7 & 34.3 & 51.1 & 24.4 & 57.1 & 37.6 \\
& old & 13.0 & 21.3 & 61.8 & 25.9 & 19.4 & 5.9 & 14.5 \\
& power law & & & 0.0 & 6.9 & & & \\
\hline
\end{tabular}

Notes: all columns have the same content as Table 3.

Table 5. Stellar populations in each of the 7 typical spectra, i.e., results of fitting typical spectra by using $15 \operatorname{SSPs}\left(Z=Z_{\odot}\right)$ from BC03.

\begin{tabular}{cc|cccc|ccc}
\hline \hline & SSP & \multicolumn{4}{|c|}{ Emission-line diagram } & \multicolumn{3}{c}{ Star-forming in $L_{\mathrm{IR}}$ bins } \\
& & star-forming & composite & LINER & Seyfert 2 & ULIG\&LIG & starburst & normal \\
\hline age & young & 39.3 & 28.0 & 4.3 & 18.3 & 47.7 & 29.7 & 45.8 \\
& intermediate & 49.6 & 59.5 & 50.0 & 59.3 & 42.2 & 63.3 & 53.5 \\
& old & 11.1 & 12.5 & 36.9 & 18.4 & 10.1 & 7.0 & 0.7 \\
& power law & & & 8.8 & 4.0 & & & \\
\hline
\end{tabular}

Notes: all columns have the same content as Table 3.

\subsection{The spectral synthesis for typical spectra by using SSPS of $Z_{\odot}$}

Up to now, we have been working on the combined spectra of galaxies in the related classes. But it would be important to indicate the variation in our results when a single spectrum instead of a synthetic average spectral energy distribution (SED) is used. Thus, we took one typical SED in each of the 7 classes and re-do all the fits. This time we adopted 15 SSPs with $Z=Z_{\odot}$ following Sect. 3.4. We did not expect much differences from the results obtained above. The results are given in Table 5 and Figs. 5, 6. It is true there are no obvious differences from the results given in Table 3, i.e., the case of $Z=Z_{\odot}$ for the combined spectra. Also there is not much difference between these and the results in Tables 2 and 4. Again, these could confirm our robust analyses of stellar populations of the sample galaxies.

\subsection{The spectral synthesis by using spectra of star clusters}

In this section, we use 15 spectra of star clusters with different ages and metallicities given in Bica \& Alloin (1986a,b) to re-fit the combined spectra of the subgroup galaxies. The resolutions of the two sets of spectra have been matched. The results are given in Figs. 7 and 8 for the different spectral classes and infrared luminosity bins. We can see that after changing our bases into spectra of the star cluster, the area around $\mathrm{H} \beta$ obtains a much better fit.

We then arranged all bases into 4 age-bins: old populations $\geq 10^{10} \mathrm{yr}$, young populations $\leq 5 \times 10^{8} \mathrm{yr}$, intermediate age populations $5 \times 10^{8}-10^{10} \mathrm{yr}$, and H II. We list the stellar population fractions of each age bin in all subsamples in Table 6. The same, these numbers are the percent fractions. We can see that LINERs present an overwhelming fraction from old populations, the starforming galaxies have younger populations than the others, and the significance of young $\left(\leq 5 \times 10^{8}\right)$ populations decreases from star-forming, composite, Seyfert 2, to LINER. These are consistent with the results obtained by using the BC03's SSP. As to the star-forming galaxies in different $L_{\mathrm{IR}}$ bins, the results are consistent with those from the BC03's SSP as well.

In addition, we also used the SSPs of Vazdekis/Miles (Vazdekis 1999; Sánchez-Blázquez et al. 2006; Koleva et al. 2008) instead of those of BC03 to re-fit the spectra, and the results are consistent with those from the BC03's SSP.

\section{The relations of some property parameters from emission lines, absorption lines, and continua}

In this section, we investigate the relations of some property parameters obtained from spectral synthesis and the emission lines or continua measurements.

The outputs of STARLIGHT are divers, and we discuss some of them in this paper, i.e., the $V$-band stellar extinction $A_{V}^{*}$, the light-weighted mean stellar age $\left\langle\log t_{*}\right\rangle_{L}$ (Eq. (7)), and the mass-weighted mean stellar metallicity $\left\langle Z_{*}\right\rangle_{M}$ (Eq. (8)):

$$
\begin{aligned}
& \left\langle\log t_{*}\right\rangle_{L}=\sum_{j=1}^{N_{*}} x_{j} \log t_{*, j}, \\
& \left\langle Z_{*}\right\rangle_{M}=\sum_{j=1}^{N_{*}} \mu_{j} Z_{*, j}
\end{aligned}
$$



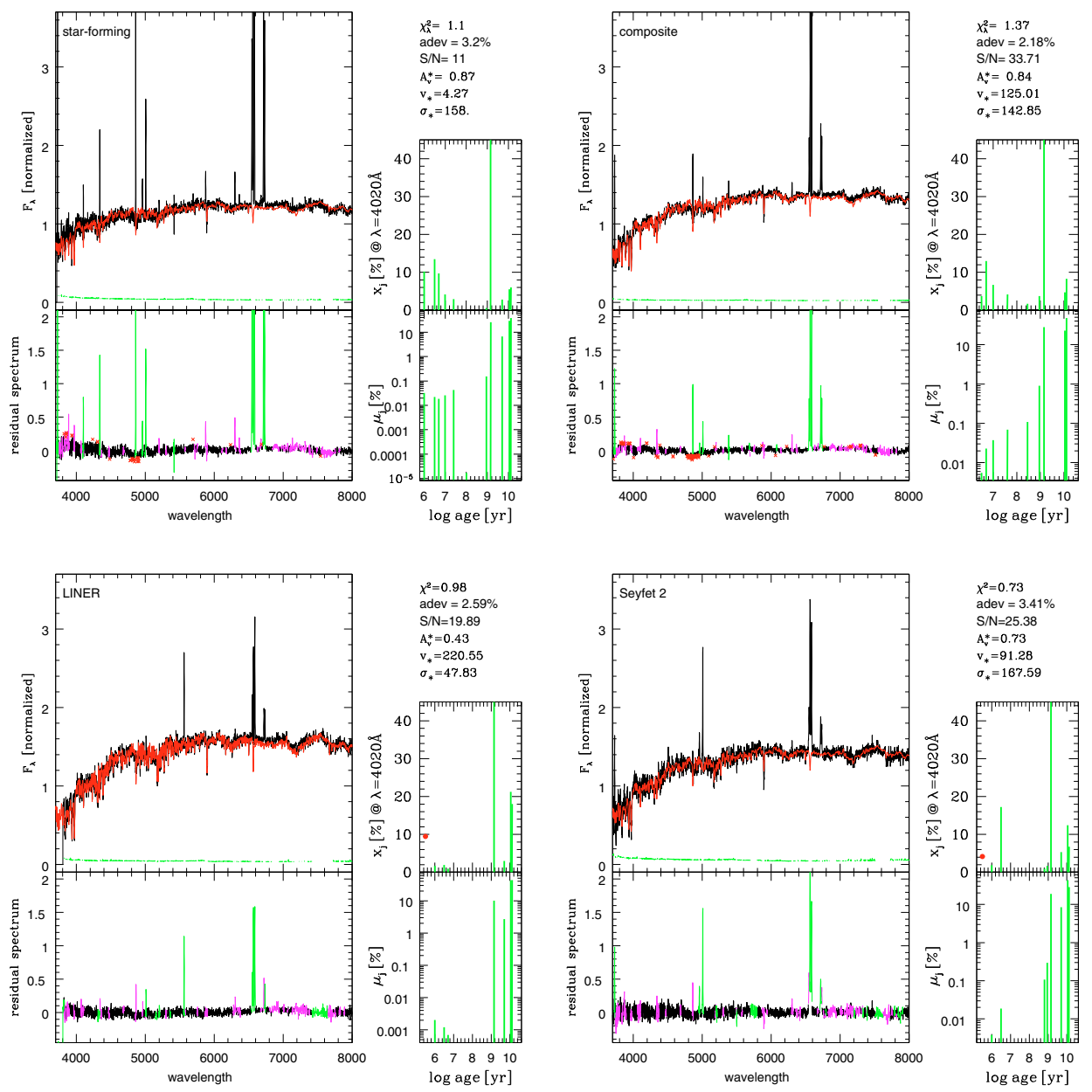

Fig. 5. Spectral synthesis of 4 typical spectra of 4 spectral classes by using 15 SSPs $\left(Z=Z_{\odot}\right)$ from BC03 as bases: top-left four panels are for star-forming galaxy (PID-MJD-FID: 0310-51990-579), top-right four panels are for composite galaxy (0499-51988-631), bottom-left four panels are for LINER (0845-52381-633), and bottom-right four panels are for Seyfert 2 (0933-52642-185). All symbols are the same as in Fig. 3.
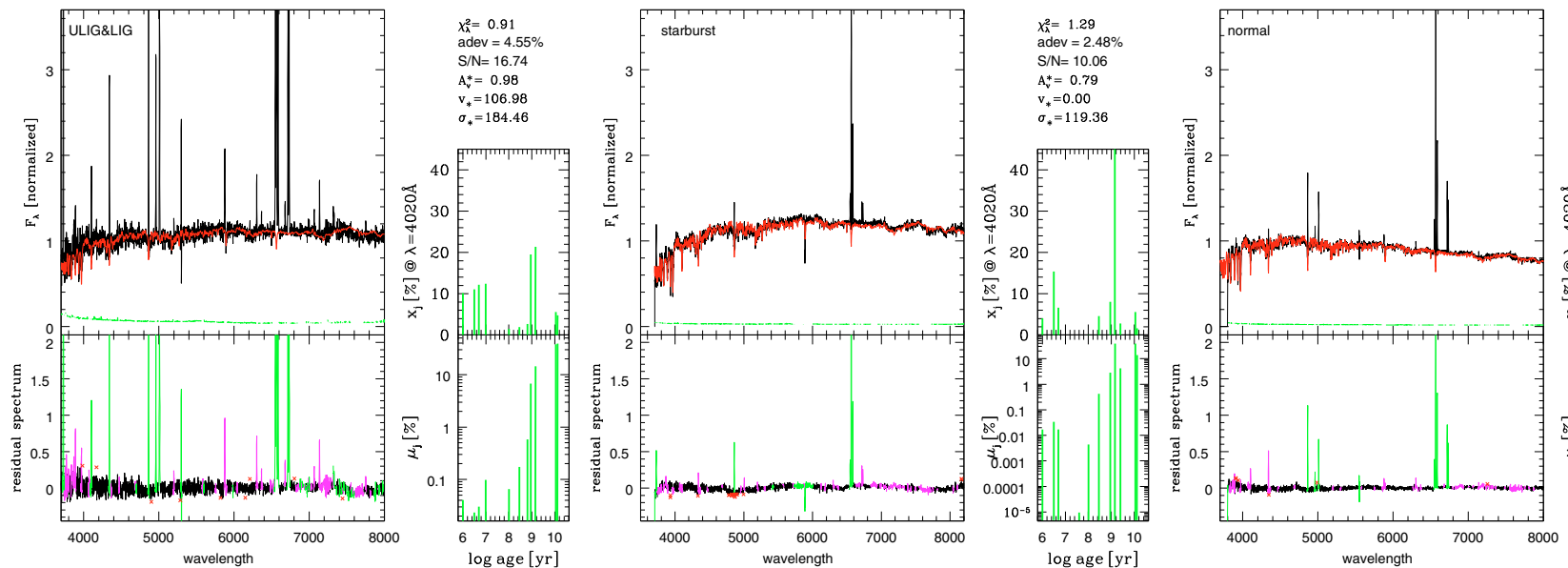

\section{$\chi_{x}^{2}=0.89$
adev $=1.82 \%$} adev $=1.82 \%$
$\mathrm{~S} / \mathrm{N}=8.56$ $\mathrm{A}^{*}=0.44$
$\mathrm{~V}=176.39$ $\mathrm{v}_{+}=176.39$
$\sigma_{*}=65.41$

Fig. 6. Spectral synthesis of 3 typical spectra in each $L_{\mathrm{IR}}$ bin of star-forming galaxies by using $15 \mathrm{SSPs}\left(Z=Z_{\odot}\right)$ from $\mathrm{BC} 03$ as bases: the left four panels are for the LIG (PID-MJD-FID: 0305-51613-093), the middle four panels are for the starburst (1310-53033-455) and the right four panels are for the normal galaxy (0586-52023-131). All symbols are same as Fig. 3.

where the subscript $L$ or $M$ denotes a light-weighted or a massweighted average.

The MPA/JHU collaboration has put the measurements of emission lines and some property parameters for the SDSS galaxies on the MPA SDSS website ${ }^{4}$ (Kauffmann et al. 2003b;

\footnotetext{
${ }^{4}$ http://www.mpa-garching.mpg.de/SDSS/
}

Brinchmann et al. 2004; Tremonti et al. 2004, etc.). These values were obtained from the stellar-feature subtracted spectra with the spectral population synthesis code of Bruzual \& Charlot (2003). We used these emission-line flux measurements to obtain some of their property parameters, or we adopted some parameters from their catalog, e.g., $D_{n}(4000)$ and $H \delta_{A}$. 

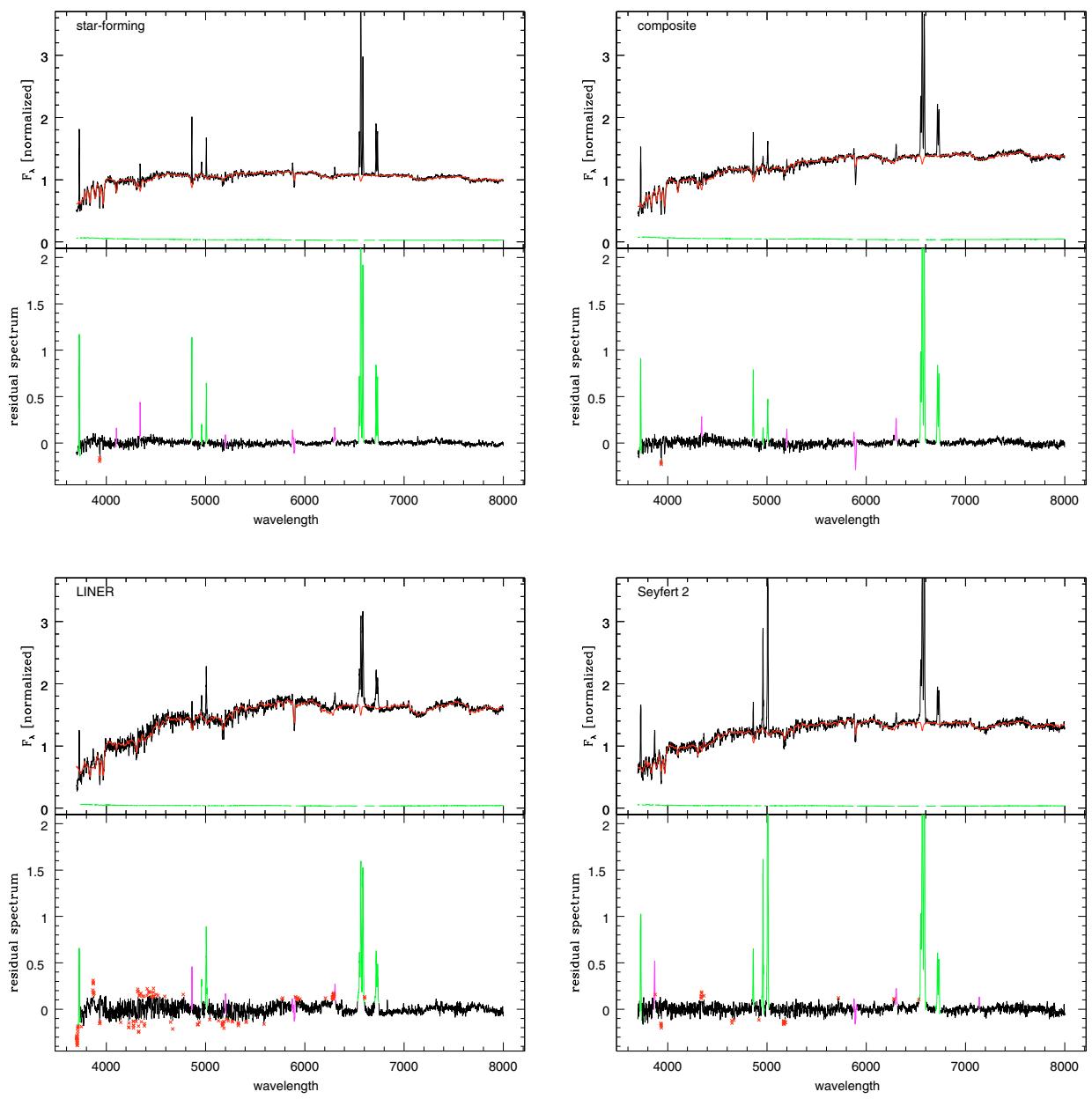

Fig. 7. Spectral synthesis of the combined spectra of 4 spectral classes by using the spectra of star clusters as bases: top-left two panels are for star-forming galaxies, top-right two panels are for composite galaxies, bottom-left two panels are for LINERs, and the bottom-right two panels are for Seyfert 2s. All symbols are the same as in the left hand columns of Fig. 3.
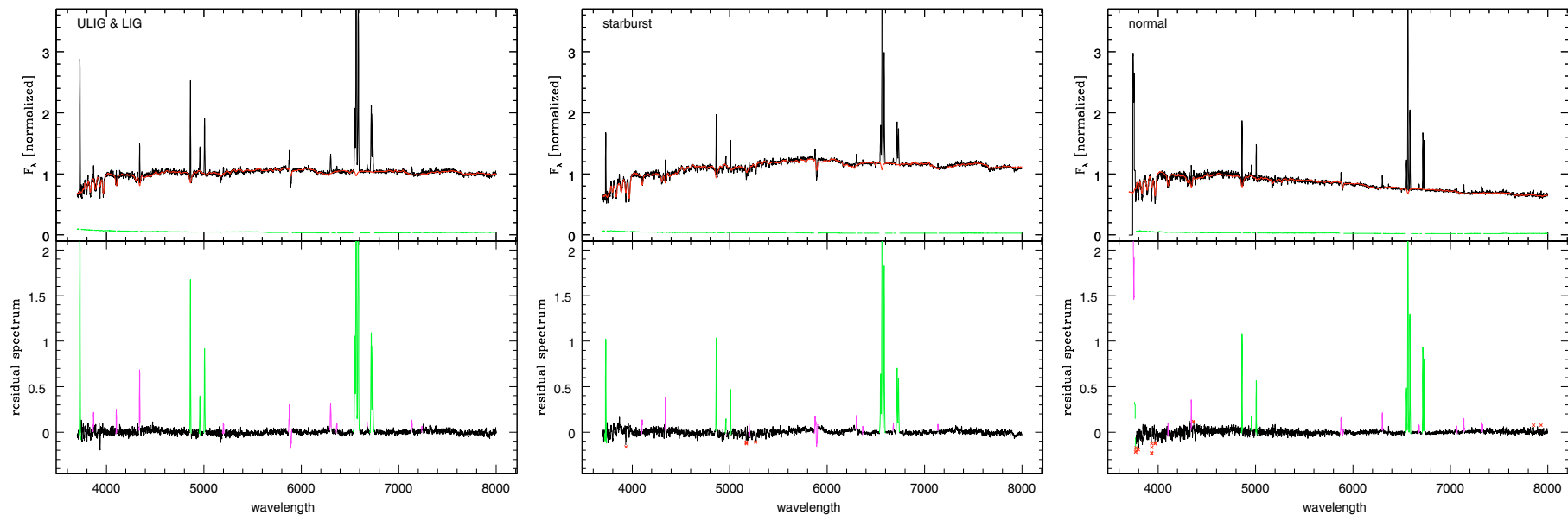

Fig. 8. Spectral synthesis of 3 combined spectra of star-forming galaxies in each $L_{\mathrm{IR}}$ bin by using the spectra of star clusters as bases: the left two panels are for ULIGs \& LIGs, the middle two panels are for starbursts, and the right two panels are for normal galaxies. All symbols are same as in the left hand columns of Fig. 3.

\subsection{Relations between stellar extinction and nebular extinction}

We estimated the nebular extinction inside the galaxies from the Balmer emission-lines ratios $\mathrm{H} \alpha / \mathrm{H} \beta$, assuming case $\mathrm{B}$ recombination, with a density of $100 \mathrm{~cm}^{-3}$ and a temperature of $10^{4} \mathrm{~K}$ (Osterbrock 1989) following the relation of

$$
\left(\frac{I_{\mathrm{H} \alpha}}{I_{\mathrm{H} \beta}}\right)_{\mathrm{obs}}=\left(\frac{I_{\mathrm{H} \alpha 0}}{I_{\mathrm{H} \beta 0}}\right)_{\mathrm{intr}} 10^{-c(f(\mathrm{H} \alpha)-f(\mathrm{H} \beta))} .
$$


Table 6. Stellar populations in each subsample, i.e., results of fitting the combined spectra by using spectra of 15 star clusters as bases.

\begin{tabular}{c|cccc|ccc}
\hline \hline Age & \multicolumn{4}{|c|}{ Emission-line diagram } & \multicolumn{3}{c}{ Star-forming in $L_{\text {IR }}$ bins } \\
& star-forming & composite & LINER & Seyfert 2 & ULIG\&LIG & starburst & normal \\
\hline young & 40.7 & 28.1 & 11.2 & 21.0 & 43.6 & 25.2 & 49.8 \\
intermediate & 19.2 & 35.5 & 0.0 & 21.7 & 16.1 & 21.4 & 0.0 \\
old & 40.1 & 36.4 & 88.8 & 52.4 & 32.8 & 53.4 & 35.0 \\
H II & 0.0 & 0.0 & 0.0 & 4.8 & 7.5 & 0.0 & 15.2 \\
\hline
\end{tabular}
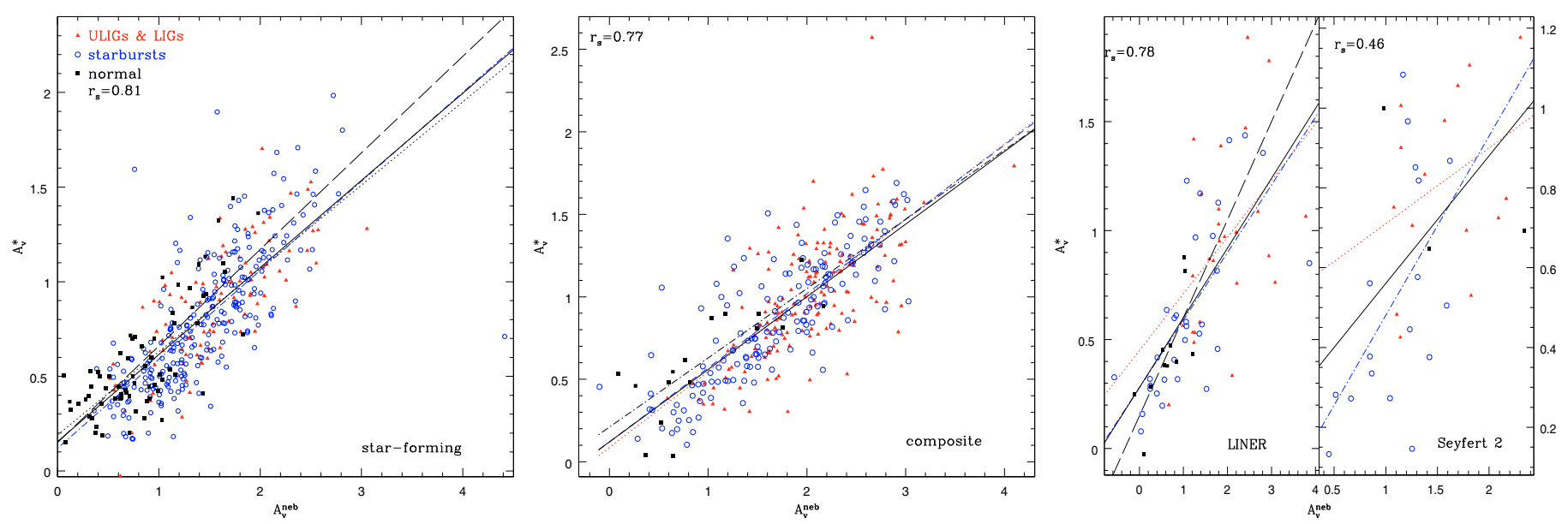

Fig. 9. Comparison between stellar and nebular extinctions for star-forming galaxies (left panel), composite galaxies (middle panel), LINERs, and Seyfert 2s (right two panels). The red triangles represent ULIGs \& LIGs (i.e. $L_{\mathrm{IR}} / L_{\odot}>10^{11}$ ), blue open circles represent starbursts (i.e. $10^{11}>L_{\mathrm{IR}} / L_{\odot}>10^{10}$ ), while black filled squares represent normal galaxies (i.e. $L_{\mathrm{IR}} / L_{\odot}<10^{10}$ ). The solid line represents a linear fit including all the sample galaxies in the plot, the dotted line represents a linear fit only including ULIGs \& LIGs, the dot-dashed line represents a linear fit only including starbursts, and the long-dashed line represents a linear fit only including normal galaxies. The Spearman-Rank Order correlation coefficient is shown in the top left corner of each panel for all the sample galaxies there.

Using the average interstellar extinction law given by Osterbrock (1989), we obtained $f(\mathrm{H} \alpha)-f(\mathrm{H} \beta)=-0.37$. We then calculated $A_{V}^{\text {neb }}$ according to Eqs. (10), (11) (Osterbrock 1989; Seaton 1979):

$\log (\mathrm{H} \alpha / \mathrm{H} \beta)_{\text {ins }}=\log (\mathrm{H} \alpha / \mathrm{H} \beta)_{\mathrm{obs}}+c \times(-0.37)$,

$A_{v}^{\text {neb }}=c \times 3.1 / 1.47$,

where $c$ is the extinction coefficient, and we set the intrinsic ratio $(\mathrm{H} \alpha / \mathrm{H} \beta)_{\text {ins }}=2.86$ for star-forming galaxies and composite galaxies, and $(\mathrm{H} \alpha / \mathrm{H} \beta)_{\text {ins }}=3.1$ for AGNs (Veilleux et al. 1995).

Comparisons between stellar extinction $A_{V}^{*}$ from our spectral synthesis and nebular extinction $A_{V}^{\text {neb }}$ calculated by using the emission-line flux measurements given in the MPA/JHU database for each subsample are presented in Fig. 9. It shows that $A_{V}^{*}$ and $A_{V}^{\text {neb }}$ are strongly correlated, and the correlation coefficients are very large.

The left hand panel of Fig. 9 presents the two $A_{V}$ values of star-forming galaxies. The linear fit including all star-forming galaxies yields $A_{V}^{*}=0.15+0.46 \times A_{V}^{\text {neb }}$, which shows that the stellar extinction is almost half of the nebular extinction. This confirms the results from other authors (Stasińska et al. 2004; Cid Fernandes et al. 2005b; Mateus et al. 2006) and accords with studies of nearby star-forming galaxies (Calzetti et al. 1994). To check whether the correlation between $A_{V}^{*}$ and $A_{V}^{\text {neb }}$ is affected by infrared luminosity $L_{\mathrm{IR}}$, we assigned different symbols to the 3 different $L_{\mathrm{IR}}$ bins, and we also plotted the corresponding linear least-square fits of them (ULIGs \& LIGs: $A_{V}^{*}=0.19+0.44 \times$ $A_{V}^{\text {neb }}$, starbursts: $A_{V}^{*}=0.12+0.47 \times A_{V}^{\text {neb }}$, and the normal galaxies: $\left.A_{V}^{*}=0.15+0.51 \times A_{V}^{\text {neb }}\right)$. We can see that these lines are close to the solid line (slope \pm 0.02 ) except for the long-dashed line. Given the distributions of stellar and nebular extinctions in the figure, we infer that the ionized gas extinction (nebular extinction) may be smaller in normal galaxies than in others, so that we get a steeper fit result for them.

Moreover, in the middle panel of Fig. 9, the linear fit including all composite galaxies yields $A_{V}^{*}=0.12+0.44 \times A_{V}^{\text {neb }}$, which infers that nebular extinction is almost twice as much as stellar extinction. This conclusion is similar to that of star-forming galaxies. Moreover, we mark different symbols for different bins of $L_{\mathrm{IR}}$ and plot the linear fit lines for these subsamples. We find that all of the three lines are close to the solid line, which is for the total sample.

Finally, in the right two panels of Fig. 9, we perform the relations between stellar extinction and nebular extinction for LINERs (left panel) and Seyfert 2s (right panel). In the left panel, the linear fit containing all LINERs yields $A_{V}^{*}=0.28+0.32 \times$ $A_{V}^{\text {neb }}$, which suggests that the ionized gas exceeds twice as much extinction as stars. It is different from the results of star-forming and composite galaxies. Furthermore, we plot three other linear fit lines for the objects in each of the $3 L_{\mathrm{IR}}$ bins in this panel, and we find that the behavior of normal galaxies is similar to normal galaxies in subsamples of star-forming and composite galaxies, while the other two subsamples perform differently. In the right hand panel, Seyfert 2s show much weaker relationship between stellar and nebular extinctions, and the Spearman-Rank Order correlation coefficient is smaller than others. Moreover, the linear fit including all 35 Seyfert 2s yields $A_{V}^{*}=0.24+0.32 \times$ $A_{V}^{\text {neb }}$. As the number of data points in Seyfert 2s' sub-sample of normal galaxies is extremely poor, we did not try linear fit to this subsample. There are very few points in AGNs, especially in Seyfert 2s, so we cannot draw definite conclusion. In total, 

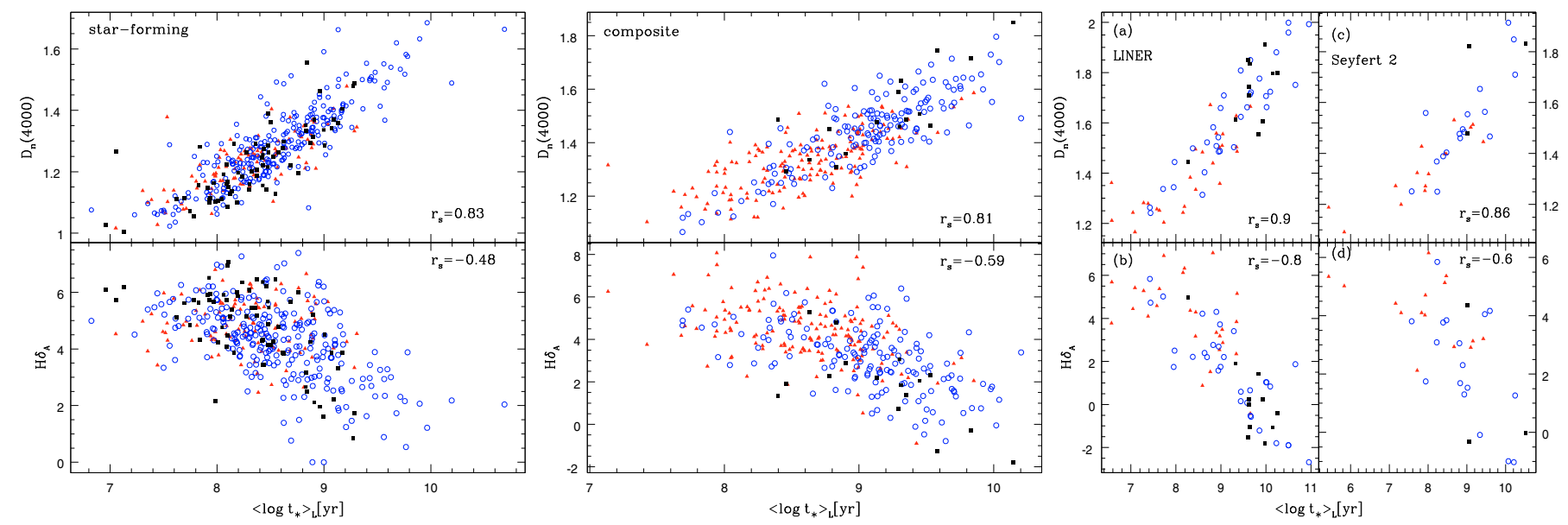

Fig. 10. The relationships of light-weighted mean age $\left\langle\log t_{*}\right\rangle_{L}$ and $D_{n}(4000), H \delta_{A}$ for star-forming galaxies (left two panels), composite galaxies (middle two panels), LINERs and Seyfert 2s (right four panels). The Spearman-Rank Order correlation coefficient are shown in each panel for all the sample galaxies there. All the symbols have the same meanings as in Fig. 9.

the ionized gas tends to suffer excess extinction twice as much as stars.

\section{2. $D_{n}(4000)$ and $H \delta_{A}$}

We also acquired two age indicators from MPA/JHU database directly: $D_{n}(4000)$ and $H \delta_{A}$.

$D_{n}$ (4000): The break at $4000 \AA$ is the strongest discontinuity in the optical spectra and is available in our sample. It was defined by Bruzual (1983) as the ratio of the average flux density in the bands 4050-4250 and 3750-3950 ̊; however, we used narrower continuum bands here (3850-3950 and 4000-4100 $)$, which was introduced by Balogh et al. (1999). In this way the index $D_{n}(4000)$ is less sensitive to reddening effects. With the increasing ages of the stellar populations of galaxies, their $D_{n}(4000)$ values will increase as well, which means the higher fraction of older populations.

$H \delta_{A}$ : Strong $H \delta$ absorption lines arise in galaxies that experienced a burst of star formation that ended $\sim 0.1-1$ Gyr ago. The peak occurs once hot $\mathrm{O}$ and $\mathrm{B}$ stars, which have weak intrinsic absorption, have terminated their evolution. The optical light from the galaxies is then dominated by late-B to early-F stars. Worthey \& Ottaviani (1997) defined an $H \delta_{A}$ index, which contains a central bandpass (4083-4122 $\AA$ in MPA/JHU database) bracketed by two pseudo-continuum band-passes.

The relationships between age indicators, $D_{n}(4000)$ and $H \delta_{A}$, and the light-weighted mean age $\left\langle\log t_{*}\right\rangle_{L}$ for each subsample are performed in Fig. 10. We also show the Spearman-rank order correlation coefficient in each panel. In the left two panels, we find there is a very strong correlation between $D_{n}(4000)$ and our light-weighted mean age for starforming galaxies. Another age indicator, $H \delta_{A}$, presents a weaker correlation (lower correlation coefficient) with mean stellar age than does $D_{n}(4000)$. The weaker correlation between $H \delta_{A}$ and the mean stellar age may be due to the fact that $H \delta_{A}$ does not monotonously vary following the evolution of the galaxy (Kauffmann et al. 2003b). Therefore, we suggest that $D_{n}(4000)$ is a good age indicator as the scatter is small and the relationship is tight in the left hand panel of Fig. 10.

For composite galaxies (middle two panels in Fig. 10), we draw a similar conclusion to that of star-forming galaxies: $D_{n}(4000)$ is a good age indicator, as the correlation coefficient is high and the diffusion is small. Although $H \delta_{A}$ performs better than that of star-forming galaxies, there are still some diffusions in the figure.

The right four panels show the results of LINERs and Seyfert 2s: the left two for LINERs and the right two for Seyfert $2 s$. In the left two we find there are strong correlations between $D_{n}(4000), H \delta_{A}$, and $\left\langle\log t_{*}\right\rangle_{L}$ for LINERs, while from the right two we find $D_{n}(4000)$ is still a good age indicator for Seyfert 2 s. In view of so few data points contained in LINERs and Seyfert 2 s, especially Seyfert 2 s, we cannot draw any absolute conclusion.

Figure 10 generally shows that the ULIGs and LIGs have relatively lower light-weighted mean ages than others, which is consistent with our results above (Sect. 3.3). In other words, we have tested all individual galaxies, so that we can confirm the robustness of the results from fitting the combined spectra.

\subsection{Nebular metallicity and mean stellar age, mean stellar metallicity}

In this section, we show the distributions between nebular metallicity $Z_{\text {neb }}$ and the light-weighted mean stellar age $\left\langle\log t_{*}\right\rangle_{L}$ (Eq. (7)), the mass-weighted mean stellar metallicity $\left\langle Z_{*}\right\rangle_{M}$ (Eq. (8)) provided by STARLIGHT. We only show the results of star-forming galaxies for two reasons, one of which is the uncertainty of the estimation of $Z_{\text {neb }}$ for composite galaxies and AGNs. Another reason is that we want to compare our results with that of Asari et al. (2007), whose sample only includes all the star-forming galaxies.

After correcting all of the useful line fluxes for extinction, we quantified the nebular metallicity $Z_{\text {neb }}$ by the oxygen abundance of the star-forming galaxies. To be consistent with Asari et al. (2007), the nebular metallicity of star-forming galaxies was obtained according to Eq. (12) where $\mathrm{O}_{3} \mathrm{~N}_{2}=$ $\log ([\mathrm{OIII}] \lambda 5007 /[\mathrm{NII}] \lambda 6583)$ (Stasińska et al. 2006; Pettini et al. 2004; Liang et al. 2006):

$\log Z_{\text {neb }}^{\mathrm{o} n 2}=\log (\mathrm{O} / \mathrm{H}) / \log (\mathrm{O} / \mathrm{H})_{\odot}=-0.14-0.25 \times \mathrm{O}_{3} \mathrm{~N}_{2}\left[Z_{\odot}\right],(12)$

where $(\mathrm{O} / \mathrm{H})_{\odot}=4.9 \times 10^{-4}$.

In Fig. 11 we show the distributions between $Z_{\text {neb }}$ and $\left\langle\log t_{*}\right\rangle_{L}$ (the left hand panel), $\left\langle Z_{*}\right\rangle_{M}$ (the right hand panel). From the left hand panel, we find there is a correlation with intermediate strength, and the correlation coefficient is 0.32 . It seems that the young stellar population makes a relatively dominant 

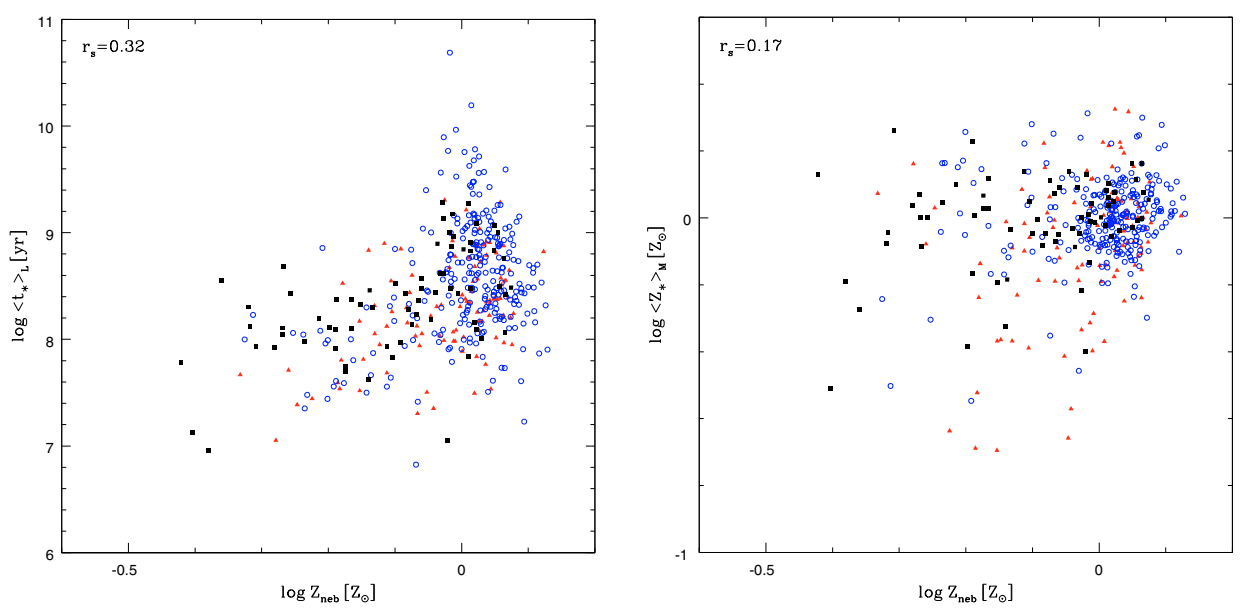

Fig. 11. The relationships between the nebular metallicity $Z_{\text {neb }}$ and the light-weighted mean age $\left\langle\log t_{*}\right\rangle_{L}$ (left panel), between $Z_{\text {neb }}$ and the massweighted mean stellar metallicity $\left\langle Z_{*}\right\rangle_{M}$ (right panel) for star-forming galaxies. The Spearman-rank order correlation coefficient are shown in each panel for all the sample galaxies there. All the symbols have the same meanings as in Fig. 9.

contribution in low metallicity galaxies. The right hand panel shows that there is a weak correlation between the mean stellar metallicity and the nebular metallicity, and the correlation coefficient is only 0.17 . The chemical enrichment histories of star and gas are correlated on the basis of simple chemical evolution scenarios, while the weakness and scatter of this relation may be due to either the disturbance from interactions/mergers and the indefinability of our calculations or the intrinsically different evolutionary phases (Asari et al. 2007). These two relations are similar to those of the total star-forming galaxies presented in the Fig. 5 in Asari et al. (2007).

\section{Summary and conclusions}

Based on a large sample (849) of local infrared galaxies selected from the cross-identification of the SDSS DR4 and IRAS PSCz, we fit the spectral absorptions and continua of the sample galaxies to study their stellar populations by using the SSPs of $\mathrm{BC} 03$ and spectra of star clusters as bases and the software STARLIGHT. The sample galaxies are divided into four or three subgroups by following their emission-line ratios or infrared luminosity. First, we investigated the effects from spectral class (star-forming, composite galaxies, LINERs, and Seyfert 2s) and infrared luminosity (ULIGs \& LIGs, starbursts, normal galaxies) on stellar populations. Second, we fit our sample galaxies with SSPs from BC03 and spectra of star clusters. Finally, we compared the stellar extinction, mean stellar age, and mean stellar metallicity derived from spectral synthesis with nebular extinction, nebular metallicity, and two age indicators, $D_{n}(4000)$ and $H \delta_{A}$, obtained from the MPA/JHU catalog.

As to the contributions to light, we found that LINERs presented the oldest stellar populations among 4 spectral classes, and the importance of young populations was decreasing from star-forming, composite galaxies, Seyfert $2 \mathrm{~s}$, to LINERs. The dominant populations of star-forming and composite galaxies are those with metallicity $Z=0.2 Z_{\odot}$, and the LINERs and Seyfert $2 \mathrm{~s}$ are more metal-rich. While ULIGs \& LIGs present the youngest populations among the star-forming galaxies in 3 infrared luminosity bins. The normal galaxies are more metal-rich than the ULIGs \& LIGs and starbursts. However, the dominant contributors to mass are all old stellar populations, and the spectral class and the infrared luminosity have no effect on this result.
The results of fittings by using spectra of star clusters as bases are consistent with those from the SSPs of $\mathrm{BC} 03$.

The stellar and nebular extinctions are correlated in all sample galaxies, and the ionized gas suffers twice as much extinction as a star. The $D_{n}(4000)$ is a much better age indicator than $H \delta_{A}$, which does not monotonously vary following the evolution of the galaxy. When we compared nebular metallicity with other parameters for star-forming galaxies, we found that the mean stellar age is correlated with nebular metallicity in an intermediate strength, and $Z_{\text {neb }}$ is weakly correlated with $\left\langle Z_{*}\right\rangle_{M}$.

Acknowledgements. We thank our referee for the valuable comments and suggestions, which helped improve this work. We thank Philippe Prugniel for very helpful discussions, and thank James Wicker for kindly correcting the text. We acknowledge the support of the Natural Science Foundation of China (NSFC) Foundation under Nos. 10403006, 10433010, 10673002, 10573022, 10333060, and 10521001, and the National Basic Research Program of China (973 Program) No. 2007CB815404,06. This work is supported by the Knowledge Innovation Program of the Chinese Academy of Sciences. The STARLIGHT project is supported by the Brazilian agencies CNPq, CAPES, and FAPESP and by the France-Brazil CAPES/Cofecub program. We thank the wonderful SDSS and IRAS database.

\section{References}

Adelman-McCarthy, J. K., Agüeros, M. A., Allam, S. S., et al. 2006, ApJS, 162, 38

Alongi, M., Bertelli, G., Bressan, A., et al. 1993, A\&AS, 97, 851

Asari, N. V., Cid Fernandes, R., Stasińska, G., et al. 2007, MNRAS, 381, 263

Baldwin, J. A., Phillips, M. M., \& Terlevich, R. 1981, PASP, 93, 5

Balogh, M. L., Morris, S. L., Yee, H. K. C., Carlberg, R. G., \& Ellingson, E. 1999, ApJ, 527, 54

Bernardi, M., Sheth, R. K., Annis, J., et al. 2003, AJ, 125, 1817

Bica, E., \& Alloin, D. 1986a, A\&A, 162, 21

Bica, E., \& Alloin, D. 1986b, A\&AS, 66, 171

Boisson, C., Joly, M., Moultaka, J., Pelat, D., \& Roos, M. S. 2000, A\&A, 357, 850

Boisson, C., Joly, M., Pelat, D., \& Ward, M. J. 2004, A\&A, 428, 373

Bonatto, C., Bica, E., Pastoriza, M. G., \& Alloin, D. 2000, A\&A, 355, 99 Bressan, A., Fagotto, F., Bertelli, G., \& Chiosi, C. 1993, A\&AS, 100, 647 Brinchmann, J., Charlot, S., White, S. D. M., et al. 2004, MNRAS, 351, 1151 Bruzual, A. G. 1983, ApJ, 273, 105

Bruzual, A. G., \& Charlot, S. 2003, MNRAS, 344, 1000

Calzetti, D., Kinney, A. L., \& Storchi-Bergmann, T. 1994, ApJ, 429, 582

Calzetti, D., Armus, L., Bohlin, R. C., et al. 2000, ApJ, 533, 682

Cao, C., Wu, H., Wang, J.-L., et al. 2006, Chinese J. Astron. Astrophys., 6, 197

Cardelli, J. A., Clayton, G. C., \& Mathis, J. S. 1989, ApJ, 345, 245

Chabrier, G. 2003, PASP, 115, 763

Charlot, S., Worthey, G., \& Bressan, A. 1996, ApJ, 457, 625

Chen, X. Y., Hao, C. N., \& Wang, J. 2008, Chinese J. Astron. Astrophys., 8, 25 
Cid Fernandes, R. 2007, in press [arXiv: astro-ph/0701902]

Cid Fernandes, R., Sodré, L., Schmitt, H. R., \& Leão, J. R. S. 2001, MNRAS, 325,60

Cid Fernandes, R., Leão, J. R. S., \& Lacerda, R. R. 2003, MNRAS, 340, 29

Cid Fernandes, R., González Delgado, R. M., Schmitt, H., et al. 2004a, ApJ, 605, 105

Cid Fernandes, R., Gu, Q., Melnick, J., et al. 2004b, MNRAS, 355, 273

Cid Fernandes, R., Delgado, R. M. G., Storchi-Bergmann, T., Martins, L. P., \& Schmitt, H. 2005a, MNRAS, 356, 270

Cid Fernandes, R., Mateus, A., Sodré, L., Stasińska, G., \& Gomes, J. M. 2005b, MNRAS, 358, 363

Cid Fernandes, R., et al. 2008, in press [arXiv:0802 . 0849]

Elbaz, D., Cesarsky, C. J., Fadda, D., et al. 1999, A\&A, 351, L37

Elbaz, D., Cesarsky, C. J., Chanial, P., et al. 2002, A\&A, 384, 848

Faber, S. M. 1972, A\&A, 20, 361

Fagotto, F., Bressan, A., Bertelli, G., \& Chiosi, C. 1994a, A\&AS, 104, 365

Fagotto, F., Bressan, A., Bertelli, G., \& Chiosi, C. 1994b, A\&AS, 105, 29

Flores, H., Hammer, F., Thuan, T. X., et al. 1999, ApJ, 517, 148

Fukugita, M., Ichikawa, T., Gunn, J. E., et al. 1996, AJ, 111, 1748

Girardi, L., Bressan, A., Chiosi, C., Bertelli, G., \& Nasi, E. 1996, A\&AS, 117, 113

González Delgado, R. M., Heckman, T., \& Leitherer, C. 2001, ApJ, 546, 845

González Delgado, R. M., Cid Fernandes, R., Pérez, E., et al. 2004, ApJ, 605, 127

Greene, J. E., \& Ho, L. C. 2005, ApJ, 627, 721

Greene, J. E., \& Ho, L. C. 2006, ApJ, 641, 117

Hammer, F., Gruel, N., Thuan, T. X., Flores, H., \& Infante, L. 2001, ApJ, 550, 570

Hammer, F., Flores, H., Elbaz, D., et al. 2005, A\&A, 430, 115

Heckman, T. M., Kauffmann, G., Brinchmann, J., et al. 2004, ApJ, 613, 109

Helou, G., Khan, I. R., Malek, L., \& Boehmer, L. 1988, ApJS, 68, 151

Joguet, B., Kunth, D., Melnick, J., Terlevich, R., \& Terlevich, E. 2001, A\&A, 380,19

Kauffmann, G., Heckman, T. M., Tremonti, C., et al. 2003a, MNRAS, 346, 1055

Kauffmann, G., Heckman, T. M., White, S. D. M., et al. 2003b, MNRAS, 341, 33

Kewley, L. J., Dopita, M. A., Sutherland, R. S., Heisler, C. A., \& Tervena, J. 2001, ApJ, 556, 121

Kewley, L. J., Groves, B., Kauffmann, G., \& Heckman, T. 2006, MNRAS, 372, 961

Koleva, M., Prugniel, Ph., Ocvirk, P., Le Borgne, D., \& Soubiran, C. 2008, MNRAS, 385, 1998

Kong, X., Charlot, S., Weiss, A., \& Cheng, F. Z. 2003, A\&A, 403, 877

Koski, A. T. 1978, ApJ, 223, 56

Le Borgne, J.-F., Bruzual, G., Pelló, R., et al. 2003, A\&A, 402, 433
Le Floc'h, E., Papovich, C., Dole, H., et al. 2005, ApJ, 632, 169

Liang, Y. C., Hammer, F., Flores, H., et al. 2004, A\&A, 423, 867

Liang, Y. C., Yin, S. Y., Hammer, F., et al. 2006, ApJ, 652, 257

Mateus, A., Sodré, L., Cid Fernandes, R., et al. 2006, MNRAS, 370, 721

Osterbrock, D. E. 1989, Astrophysics of Gaseous Nebulae and Active Galactic

Nuclei (Mill Valley: University Science Books)

Papovich, C., Rudnick, G., Le Floc'h, E., et al. 2007, ApJ, 668, 45

Pettini, M., \& Pagel, B. E. J. 2004, MNRAS, 348, L59

Raimann, D., Bica, E., Storchi-Bergmann, T., Melnick, J., \& Schmitt, H. 2000, MNRAS, 314, 295

Riffel, R., Pastoriza, M. G., Rodríguez-Ardila, A., \& Maraston, C. 2008, MNRAS, 388, 803

Sánchez-Blázquez, P., Peletier, R. F., Jiménez-Vicente, J., et al. 2006, MNRAS, 371,703

Sanders, D. B., \& Mirabel, I. F. 1996, ARA\&A, 34, 749

Saunders, W., Sutherland, W. J., Maddox, S. J., et al. 2000, MNRAS, 317, 55

Schlegel, D. J., Finkbeiner, D. P., \& Davis, M. 1998, ApJ, 500, 525

Schmitt, H. R., Bica, E., \& Pastoriza, M. G. 1996, MNRAS, 278, 965

Schmitt, H. R., Storchi-Bergmann, T., \& Cid Fernandes, R. 1999, MNRAS, 303, 173

Seaton, M. J. 1979, MNRAS, 187, 73

Smith, J. A., Tucker, D. L., Kent, S., et al. 2002, AJ, 123, 2121

Shuder, J. M., \& Osterbrock, D. E. 1981, ApJ, 250, 55

Soifer, B. T., Houck, J. R., \& Neugebauer, G. 1987, ARA\&A, 25, 187

Stasińska, G. 2006, A\&A, 454, L127

Stasińska, G., Mateus, A., Sodré, L., \& Szczerba, R. 2004, A\&A, 420, 475

Stasińska, G., et al. 2008, MNRAS, 391, L29

Storchi-Bergmann, T., Raimann, D., Bica, E. L. D., \& Fraquelli, H. A. 2000, ApJ, 544, 747

Stoughton, C., Lupton, R. H., Bernardi, M., et al. 2002, AJ, 123, 485

Strauss, M. A., Weinberg, D. H., Lupton, R. H., et al. 2002, AJ, 124, 1810

Tinsley, B. M. 1978, ApJ, 222, 14

Tremonti, C. A., Heckman, T. M., Kauffmann, G., et al. 2004, ApJ, 613, 898

Vazdekis, A. 1999, ApJ, 513, 224

Veilleux, S., \& Osterbrock, D. E. 1987, ApJS, 63, 295

Veilleux, S., Kim, D.-C., Sanders, D. B., Mazzarella, J. M., \& Soifer, B. T. 1995, ApJS, 98, 171

Wang, J. L. 2008, Chinese J. Astron. Astrophys., 8, 643

Wang, J. L., Xia, X. Y., Mao, S., et al. 2006, ApJ, 649, 722

Westera, P., Cuisinier, F., Telles, E., \& Kehrig, C. 2004, A\&A, 423, 133

Worthey, G., \& Ottaviani, D. L. 1997, ApJS, 111, 377

Zheng, X. Z., Hammer, F., Flores, H., Assémat, F., \& Pelat, D. 2004, A\&A, 421, 847

Zheng, X. Z., Dole, H., Bell, E. F., et al. 2007, ApJ, 670, 301 